\title{
$S$ Note on Transliteration AND TRANSLATION
}

All translations are my own unless otherwise indicated in the endnotes. I have used the Library of Congress system of transliteration, with occasional modifications for surnames that are more recognizable in other forms (Brodsky for Brodskii, for example). 
